\title{
Editorial: Microbial Electrogenesis, Microbial Electrosynthesis, and Electro-bioremediation
}

\author{
Sebastià Puig ${ }^{1 *}$, Ludovic Jourdin ${ }^{2}$ and Shafeer Kalathil ${ }^{3}$ \\ ${ }^{1}$ LEQUiA, Institute of the Environment, University of Girona, Girona, Spain, ${ }^{2}$ Department of Biotechnology, Faculty of Applied \\ Sciences, Delft University of Technology, Delft, Netherlands, ${ }^{3} \mathrm{Hub}$ for Biotechnology in the Built Environment, Department of \\ Applied Sciences, Faculty of Health and Life Sciences, Northumbria University, Newcastle, United Kingdom
}

Keywords: microbial electrochemical technologies, 3-D printing, electro microbiology, synthetic electrodes, kinetics modelling

\section{Editorial on the Research Topic}

\section{Microbial Electrogenesis, Microbial Electrosynthesis, and Electro-bioremediation}

OPEN ACCESS

Edited by:

Celin Acharya,

Bhabha Atomic Research Centre (BARC), India

Reviewed by:

Daniel Ross,

National Energy Technology Laboratory (DOE), United States

Pratyoosh Shukla

Banaras Hindu University, India

*Correspondence:

Sebastià Puig

sebastia.puig@udg.edu

Specialty section:

This article was submitted to

Microbiotechnology,

a section of the journal

Frontiers in Microbiology

Received: 16 July 2021

Accepted: 20 August 2021

Published: 13 September 2021

Citation:

Puig S, Jourdin L and Kalathil S (2021) Editorial: Microbial Electrogenesis,

Microbial Electrosynthesis, and Electro-bioremediation.

Front. Microbiol. 12:742479.

doi: 10.3389/fmicb.2021.742479
Microbial electrochemical technology (MET) is a bioelectrochemical platform with the potential to overcome a broad range of environmental issues (i.e., global energy, climate crises and water scarcity, among others). This emerging field of research represents an interdisciplinary approach that combines the strengths of microbial catalysis, synthetic electrodes, and analytical techniques for the production of energy and chemicals from waste carbon. The interest in METs has been growing over the years. As an example, the number of papers published in 2020 for the keywords of this Research Topic were 'Microbial Electrogenesis (10 papers; 9 in 2017)', 'Microbial Electrosynthesis ( 83 papers; 61 in 2017)', and 'Electro-bioremediation (51 papers; 25 in 2017)' according to the Scopus database (July 29th, 2021).

Of particular interest are electroactive microbes with the naturally-evolved ability to electrically interact with insoluble electron acceptors or donors (metal oxides/electrodes) for anaerobic respiration or fermentation, which promises broad applications in microbial fuel cells (Lovley and Holmes, 2021), microbial electrosynthesis (MES; Dessì et al., 2021) and electro-bioremediation (Wang et al., 2020). A key limitation of METs arises from the imperfect integration of microbes with electrode materials which limits the power output, treatment capacity, or volumetric productivity depending on the application (Domínguez-Garay et al., 2018; Fang et al., 2020; Jourdin et al., 2020). Therefore, the nexus of breakthroughs lie in the electrode architecture and microbial interfaces.

The articles published in this Research Topic represent a step forward in the development of METs. For instance, Erben et al. studied the influence of local acidification on the current production of Shewanella oneidensis MR-1/electrode composites using various buffer concentrations from 10 to $40 \mathrm{mM}$. The results suggest that proton transport associated with electron transfer dictates the rate of extracellular electron transport in S. oneidensis MR-1 in agreement with a previous report (Okamoto et al., 2017). This study concluded that current production was dictated by the chemical (micro)-environment (i.e., local acidification) and the anode material to promote biofilm growth. The electrospun anode materials proposed in this study could provide a solution for high current densities by facilitating biofilm formation while minimising media costs.

MES systems are plagued with poor-performing electrodes that limit microbial activity (Prévoteau et al., 2020; Dessì et al., 2021; Jourdin and Burdyny, 2021). Significant efforts have been given to develop improved cathodes for MES. Kracke et al. propose the use of 3D-printed 
custom electrodes to fine-tune $\mathrm{H}_{2}$ delivery during MES. The idea behind this is to integrate a $3 \mathrm{D}$ fabricated carbon aerogel cathode plated with nickel-molybdenum and Methanococcus maripaludis for electromethanogenesis. Specifically, the nickelmolybdenum coating catalysed the $\mathrm{H}_{2}$ evolution reaction catalysts that provided $\mathrm{H}_{2}$ to $M$. maripaludis while the $3 \mathrm{D}$ structure enhanced the catalytically active surface area. These modifications could mitigate the effects of bubble formation and local $\mathrm{pH}$ gradients within the boundary layer, hence overcoming some key constraints on in situ electron delivery in MES.

Breakthrough understanding of the process limiting steps in MES can be achieved using computational modelling tools. Cabau-Peinado et al. developed a general framework for modelling microbial kinetics in biofilm-driven MES of carboxylates (e.g., acetate, n-butyrate, and n-caproate) from $\mathrm{CO}_{2}$. The model was fitted and validated using experimental data from different research groups. The results indicate significant substrate limitation (as $\mathrm{CO}_{2}$ dissolved concentration) in existing MES systems and suggest that operating MES in continuous mode (as constant $\mathrm{CO}_{2}$ sparging and continuous flow of fresh medium) enhances microbial growth and allows higher current densities to be achieved.

Another interesting approach is the use of electro fermentation to overcome the metabolic limitations of fermentative pathways. Application of current can alter metabolic pathways in fermentative microbes and can yield energetically unfavourable products which are not commonly produced by traditional fermentation reactions

\section{REFERENCES}

Dessì, P., Rovira-Alsina, L., Sánchez, C., Dinesh, G. K., Tong, W., Chatterjee, P., et al. (2021). Microbial electrosynthesis: towards sustainable biorefineries for production of green chemicals from $\mathrm{CO}_{2}$ emissions. Biotechnol. Adv. 46:107675. doi: 10.1016/j.biotechadv.2020.107675

Domínguez-Garay, A., Quejigo, J. R., Dörfler, U., Schroll, R., and Esteve-Núñez, A. (2018). Bioelectroventing: an electrochemical-assisted bioremediation strategy for cleaning-up atrazine-polluted soils. Microb. Biotechnol. 11, 50-62. doi: 10.1111/1751-7915.12687

Fang, X., Kalathil, S., Divitini, G., Wang, Q., and Reisner, E. (2020). A three-dimensional hybrid electrode with electroactive microbes for efficient electrogenesis and chemical synthesis. Proc. Natl. Acad. Sci. U.S.A. 117, 5074-5080. doi: 10.1073/pnas.1913463117

Jourdin, L., and Burdyny, T. (2021). Microbial electrosynthesis : where do we go from here ? Trends Biotechnol. 39, 359-369. doi: 10.1016/j.tibtech.2020.10.014

Jourdin, L., Sousa, J., Stralen, N., van, and Strik, D. P. B. T. B. (2020). Technoeconomic assessment of microbial electrosynthesis from $\mathrm{CO}_{2}$ and/or organics: an interdisciplinary roadmap towards future research and application. Appl. Energy 279:115775. doi: 10.1016/j.apenergy.2020.115775

Logan, B. E., Rossi, R., Ragab, A., and Saikaly, P. E. (2019). Electroactive microorganisms in bioelectrochemical systems. Nat. Rev. Microbiol. 17, 307-319. doi: 10.1038/s41579-019-0173-x

Lovley, D. R., and Holmes, D. E. (2021). Electromicrobiology: the ecophysiology of phylogenetically diverse electroactive microorganisms. Nat. Rev. Microbiol. doi: 10.1038/s41579-021-00597-6

Okamoto, A., Tokunou, Y., Kalathil, S., and Hashimoto, K. (2017). Proton transport in the outer-membrane flavocytochrome complex limits the rate of extracellular electron transport. Angew. Chemie Int. Ed. 56, 9082-9086. doi: 10.1002/anie.201704241
(Schievano et al., 2016; Logan et al., 2019). Isipato and colleagues proposed electro fermentation to enhance propionate production via controlling lactate fermentation and to recycle the resulting $\mathrm{CO}_{2}$ into acetate, thus increasing the volatile fatty acid yield and reducing the addition of chemicals for $\mathrm{pH}$ control (Isipato et al.).

In summary, we believe that the collection of articles included in this Research Topic can pave the way for the development of a high-performing bioelectrochemical platform for sustainable power generation and chemical synthesis.

\section{AUTHOR CONTRIBUTIONS}

All authors listed have made a substantial, direct and intellectual contribution to the work, and approved it for publication.

\section{FUNDING}

SP is a Serra Húnter Fellow (UdG-AG-575) and acknowledges the funding from the ICREA Acadèmia award and the Spanish Ministry of Science (RTI2018-098360-B-I00). LEQUIA (http:// www.lequia.udg.edu/) has been recognized as a consolidated research group by the Catalan Government (2017-SGR-1552). LJ acknowledges the funding from Shell and a PPP-allowance from Top Consortia for Knowledge and Innovation (TKI's), of the Ministry of Economic Affairs and Climate in the context of the TU Delft e-Refinery Institute. SK acknowledges Research England's Expanding Excellence in England (E3) Fund.

Prévoteau, A., Carvajal-Arroyo, J. M., Ganigué, R., and Rabaey, K. (2020). Microbial electrosynthesis from $\mathrm{CO}_{2}$ : forever a promise? Curr. Opin. Biotechnol. 62, 48-57. doi: 10.1016/j.copbio.2019.08.014

Schievano, A., Pepé Sciarria, T., Vanbroekhoven, K., De Wever, H., Puig, S., Andersen, S. J., et al. (2016). Electro-fermentation-merging electrochemistry with fermentation in industrial applications. Trends Biotechnol. 34, 866-878. doi: 10.1016/j.tibtech.2016.04.007

Wang, X., Aulenta, F., Puig, S., Esteve-Núñez, A., He, Y., Mu, Y., et al. (2020). Microbial electrochemistry for bioremediation. Environ. Sci. Ecotechnol. 1, 100013. doi: 10.1016/j.ese.2020.10 0013

Conflict of Interest: The authors declare that the research was conducted in the absence of any commercial or financial relationships that could be construed as a potential conflict of interest.

Publisher's Note: All claims expressed in this article are solely those of the authors and do not necessarily represent those of their affiliated organizations, or those of the publisher, the editors and the reviewers. Any product that may be evaluated in this article, or claim that may be made by its manufacturer, is not guaranteed or endorsed by the publisher.

Copyright (c) 2021 Puig, Jourdin and Kalathil. This is an open-access article distributed under the terms of the Creative Commons Attribution License (CC BY). The use, distribution or reproduction in other forums is permitted, provided the original author(s) and the copyright owner(s) are credited and that the original publication in this journal is cited, in accordance with accepted academic practice. No use, distribution or reproduction is permitted which does not comply with these terms. 